\title{
Dynamic Analysis and Degenerate Hopf Bifurcation-Based Feedback Control of a Conservative Chaotic System and Its Circuit Simulation
}

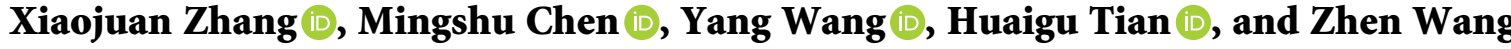 \\ Shaanxi International Joint Research Center for Applied Technology of Controllable Neutron Source School of Science, \\ Xijing University, Xi'an 710123, China \\ Correspondence should be addressed to Zhen Wang; williamchristian@163.com
}

Received 4 February 2021; Accepted 19 May 2021; Published 30 May 2021

Academic Editor: Shijian Cang

Copyright (C) 2021 Xiaojuan Zhang et al. This is an open access article distributed under the Creative Commons Attribution License, which permits unrestricted use, distribution, and reproduction in any medium, provided the original work is properly cited.

\begin{abstract}
A novel conservative chaotic system with no equilibrium is investigated in this study. Various dynamics such as the conservativeness, coexistence, symmetry, and invariance are presented. Furthermore, a partial-state feedback control scheme is proposed, and the stable domain of control parameters is analyzed based on the degenerate Hopf bifurcation. In order to verify the numerical simulation analysis, an analog circuit is designed. The simulation results show that the output of the analog circuit system can reproduce the numerical simulation results and verify the correctness of the theoretical analysis.
\end{abstract}

\section{Introduction}

Since Lorenz first found the chaotic attractor in the quadratic polynomial ODEs in 1963 [1], many typical chaotic attractors including Rössler attractor [2], Chen and Ueta attractor [3], and Lü attractor [4] were discovered in many nonlinear systems $[5,6]$ in the following decades. However, the attractors of most of them are Shilnikov type, and the corresponding systems have saddle-foci equilibria [7]. In 2011, Leonov first discovered the hidden attractors in Chua's chaotic circuit [8]; unlike the Shilnikov-type attractors, these hidden attractors are with a basin of attraction which does not contain neighborhoods of equilibria and cannot be determinated by the Shilnikov condition. In the last decade, the systems with hidden attractors mainly include chaotic systems without equilibrium [9], with infinite equilibrium $[10,11]$, and with only one stable equilibrium [12-17]. Although many chaotic systems with hidden attractors have been proposed, most of them are mainly studied by numerical simulation, and the main dynamic behaviors studies are focused on the local structures, while the studies on the global dynamics are relatively rare; hence, it is necessary to study the global dynamics and bifurcation of chaotic systems with hidden attractors to reveal the mechanism of chaos.

In recent years, in the field of hidden attractors, many researchers have paid attention to the coexisting hidden attractors which have more complex hidden characteristics than the ordinary hidden attractors. A modified Chua's circuit is implemented using a 5-segment piecewise-linear Chua's diode, and the coexistence of hidden attractors with other three attractors is presented [18]. In 2018, V.T. Pham and his coauthors presented a simple fractional-order 3D chaotic system without equilibrium and explored the coexistence of various attractors [19]. Li [20] constructed a 4D simplified Lorenz system which can generate a variety of coexisting hidden attractors, including the symmetric hidden attractors and the hyperchaotic attractors. It should be noted that most of the above systems are dissipative, so how to construct a conservative system with hidden attractors has become a hot issue in chaos theory. Moreover, according to Liouville's theorem, for these conservative dynamical systems, the volume of phase space is conservative and the flow is incompressible. Allan and Michael constructed a system with coexisting quasiperiodic and coexisting chaotic flows in 
2013 [21]. 3D/4D conservative chaotic systems were constructed by Cang [22, 23]; meanwhile, the dynamics and conservative flows were also researched. Furthermore, conservative chaotic mapping has been revealed in $[24,25]$.

In addition, there are coexisting chaotic flows in conservative chaotic systems, which may be chaotic flows coexisting with torus [26] or one or more limit cycles [27-31] and so on. What causes the coexisting chaotic flows in chaotic systems? While some are caused by offset boosting, Li explored a new regime of chaotic flow, in which one of the variables has the freedom of offset boosting, and a different symmetry was obtained by using a new boosting controller, thereby retaining any existing multistability [32]. Some of them are caused by the symmetry of conditions, different initial values will produce different chaotic flows, and the conditional symmetry may also produce coexisting symmetric chaotic flows $[33,34]$. Some are from the structure of hypogenetic Jerk [35]. Such nonequilibrium conservative chaotic systems with coexisting symmetric chaotic flows are very important in engineering applications and may have problems, so it is necessary to study the types and characteristics of their coexisting chaotic flows. Based on the complex characteristics, the conservative coexisting chaotic flows have been applied to many engineering systems, such as tank circuit, chaotic communication, image, video and audio encryption [36], control system, and pseudorandom number generator [37].

On the other hand, the Hopf bifurcation of nonlinear dynamical systems has attracted more and more attention, which is the critical point of stability switching and periodic solution of a system. In order to eliminate saddle node bifurcation or reduce the generated amplitude, it is necessary to design a reasonable nonlinear controller, in which earliest can be traced back that Abed studied the control of Hopf bifurcation [38]. Subsequently, a general explicit formula is derived [39]; this method does not increase the size of the system and can be used to delay (determine) existing forks or change the stability of the fork solution. Ding proposed a time-delayed feedback controller in order to control the fractional-order memristor based chaotic system with timedelay [40]. Now, the work of bifurcation control involves the engineering fields such as aviation [41], electrical machinery [42], and bioengineering [43]. The main feature of conservative systems is that they have chaotic flow instead of the attractor, which means that these systems have complex dynamic characteristics. Thus, it is necessary to analyze the chaotic dynamics of conservative systems. Motivated by this, a new conservative chaotic system with no equilibrium is introduced, and the dynamic characteristics of this new system are analyzed in detail. A feedback control scheme based on the degenerate Hopf bifurcation method is proposed. Finally, the simulation results are illustrated by using the analog circuit.

The main contributions of this study lie in the following aspects:

(1) A novel conservative chaotic system with no equilibrium is investigated. Since the investigated system does not have equilibrium, it is very challenging to analyze its dynamics. We analyzed the conservativeness, coexistence, symmetry, and invariance.

(2) A new partial-state feedback control by using onedimensional state for the investigated conservative chaotic system is proposed. Compared with the fullstate feedback control methods, the proposed scheme is required to measure less states and provides a simpler controller structure. Thus, the proposed partial-state feedback control scheme can be easily applied in the practical engineering. Furthermore, degenerate Hopf bifurcation was analyzed to illustrate the parametric stability range of the state feedback control system.

The structure of this study is as follows: in Section 2, the conservative chaotic system and the dynamics analysis are presented, including the conservativeness, the symmetry and invariance, and coexisting chaotic behaviors. In Section 3, a partial-state feedback control for the investigated system is proposed, and the stable domain of control parameters is analyzed based on the degenerate Hopf bifurcation method. In Section 4, the numerical simulation results are illustrated by using the analog circuit. Finally, the conclusion is presented in Section 5.

\section{Dynamics Analysis}

The new conservative chaotic system is given as follows:

$$
\begin{aligned}
& \dot{x}=y, \\
& \dot{y}=-x-y z, \\
& \dot{z}=a-b x^{2}+c y^{2} .
\end{aligned}
$$

Let $\dot{x}=0, \dot{y}=0, \dot{z}=0$, system (1) has a line equilibrium $(0,0, z)$ when $a=0$ or with no equilibrium when $a \neq 0$. In order to further study the dynamics of system (1), the numerical simulation analysis will be carried out in the following subsections.

2.1. Conservativeness. The conservativeness of system (1) can be testified by the divergence

$$
\nabla V=\frac{\partial \dot{x}}{\partial x}+\frac{\partial \dot{y}}{\partial y}+\frac{\partial \dot{z}}{\partial z}=-z(t)
$$

Obviously, the conservativeness is not obvious since the dissipation is given by the time-averaged value of $-z(t)$ along the trajectory. Following the definition, the average value of a variable $s(t)$ is given by $\bar{s}(t)=\lim _{t \rightarrow \infty}\left(\int_{t_{0}}^{t} s(t) \mathrm{d} t / t-t_{0}\right)$, and the average of $z(t)$ of system (1) with special parameters (from Table 1, we can see that system (1) is chaotic for these parameters.) is shown in Figure 1. It can be seen that the average values of $z(t)$ for system (1) will be zero.

In fact, when $b=0, c=1$, system (1) is a special case of the Nose-Hoover oscillator [44], which is invariant to the transformation $t \longrightarrow-t$. Furthermore, we can provide a positive definite energy function which has a quadratic form 
TABle 1: Motion states of system (1) for different values of the parameters $a, b$ under $c=1$ with initial value $(0,5,0)$.

\begin{tabular}{|c|c|c|c|c|}
\hline Parameter & Lyapunov exponents & Lyapunov dimension & Motion states & Phase portrait \\
\hline$a=-5, b=0$ & $(0.003,0,-0.003)$ & 3 & Rotationally symmetric chaotic flow & Figure 2(a) \\
\hline$a=-4, b=0$ & $(0.003,0,-0.003)$ & 3 & Rotationally symmetric chaotic flow & Figure 2(b) \\
\hline$a=-0.8, b=0$ & $(0.013,0,-0.013)$ & 3 & Symmetric pair of chaotic flow & Figure $2(\mathrm{c})$ \\
\hline$a=-0.5, b=0$ & $(0.003,0,-0.003)$ & 3 & Rotationally symmetric chaotic flow & Figure $2(\mathrm{~d})$ \\
\hline$a=-0.2, b=0$ & $(0.004,0,-0.004)$ & 3 & Symmetric pair of chaotic flow & Figure $2(\mathrm{e})$ \\
\hline$a=-0.15, b=0$ & $(0.003,0,-0.003)$ & 3 & Symmetric pair of chaotic flow & Figure 2(f) \\
\hline$a=-6.17, b=-1$ & $(0.004,0,-0.005)$ & 2.8 & Rotationally symmetric chaotic flow & Figure $2(\mathrm{~g})$ \\
\hline$a=-4.5, b=-1$ & $(0,-0.37,-0.95)$ & 1 & Symmetric limit cycle & Figure $2(\mathrm{~h})$ \\
\hline$a=-0.8, b=-1$ & $(0,-0.01,-26.94)$ & 1 & Symmetric pair of limit cycles & Figure 2(i) \\
\hline
\end{tabular}

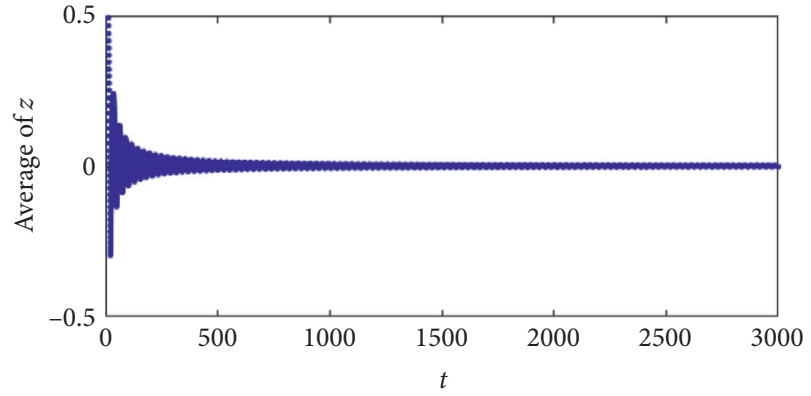

(a)

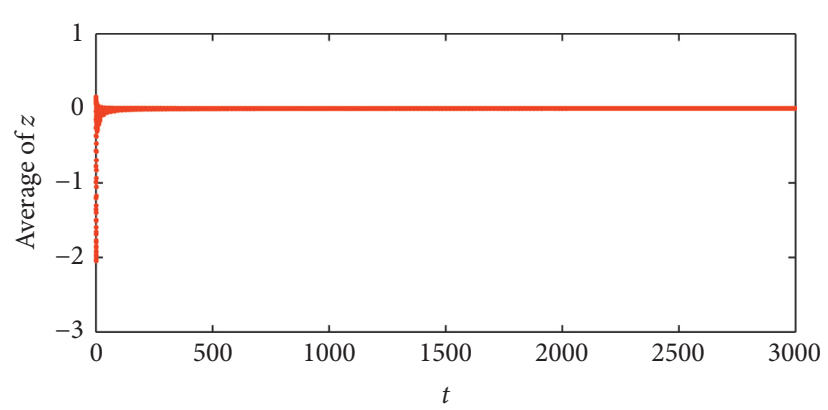

(b)

Figure 1: Time-averaged value of $z(t)$ for system (1) with different parameters $a, b, c$. (a) $a=0.2, b=0, c=1$. (b) $a=-4, b=0, c=1$.

$$
H=\frac{1}{2}\left(x^{2}+y^{2}+z^{2}\right)
$$

Taking the time derivative of $H$, we have

$$
\dot{H}=x \dot{x}+y \dot{y}+z \dot{z}=a z \text {. }
$$

If the average of $z$ is zero, we know that the energy of system (1) is invariable. Therefore, the system has a conservative nature (Table 1 ).

\subsection{Symmetry and Invariance. It is obviously that system (1)} is invariant under the transformation $(x, y, z) \longrightarrow(-x,-y, z)$, i.e., system (1) is symmetrical about the coordinate axis $z$. Interestingly, it is time reversible with LEs that are symmetric about zero for $b=0$ (Table 1).

When the initial value is $(0,5,0)$, the parameter $c$ is fixed and the parameters $a$ and $b$ are changed, and the phase diagrams with different parameters are shown in Figure 2 and summarized in Table 1. It can be seen from Figure 2 that when the Lyapunov dimension is 3, there are chaotic motions, but there are no obvious chaotic attractors.

From Table 1, when the Lyapunov dimension equals the system dimension and the sum of finite-time local Lyapunov exponents is approximately zero, system (1) has a conservative solution (Figures 2(a)-2(f)). In addition, system (1) also has symmetric limit cycles (Figures 2(h)2(i)). Moreover, when the values of parameters are changed, the classic butterfly attractor is broken into a symmetric pair of strange attractors, or shrinks into a small attractor basin, which is intertwined with a pair of symmetrical limit cycle basins, resulting in symmetry broken
$[45,46]$. Under the different initial values $(0,5,0)$ and $(0,-5,0)$, when $a=-0.8, b=0, c=1$, we can find coexistence of symmetric pair of chaotic flow as shown in Figure 2(c). The two chaotic domains are represented by red and light blue, respectively. Similarly, in Figure 2(e), $a=-0.2$, and it shows a typical symmetric pair of chaotic flow in the region, where $a$ is relatively small. As $a$ increases, a new pair of nearly touching chaotic flows appears, as in Figure 2(f) $(a=-0.15)$.

2.3. Coexistence. From Section 2.2, we can see that system (1) has some chaotic flows. Moreover, since the sum of Lyapunov exponents is approximately zero for system (1) with special parameters, the conservative solution and chaotic flows will coexist in this system (Figure 3(a), chaotic flow coexists a torus). Indeed, there is a limit cycle for some initial points from $a-b x^{2}+c y^{2}=0, z=0$ under $a<0$, $-b=c=1$. Following these conditions, if an initial point starts from a limit cycle $x^{2}+y^{2}=-a, z=0$, we have $\dot{z}=0$, and the variable $z$ will be fixed. Hence, when $z \equiv 0$, system (1) will become

$$
\begin{aligned}
& \dot{x}=y, \\
& \dot{y}=-x .
\end{aligned}
$$

The system (5) is a Hamiltonian system (conservative system). All orbits starting from $x^{2}+y^{2}=-a$ will stay in this circle. So we claim that system (1) will have a limit cycle (green cycle shown in Figures 3(b)-3(d)) which coexists with other attractors (including chaotic flow) under the condition of $a<0,-b=c=1$. 


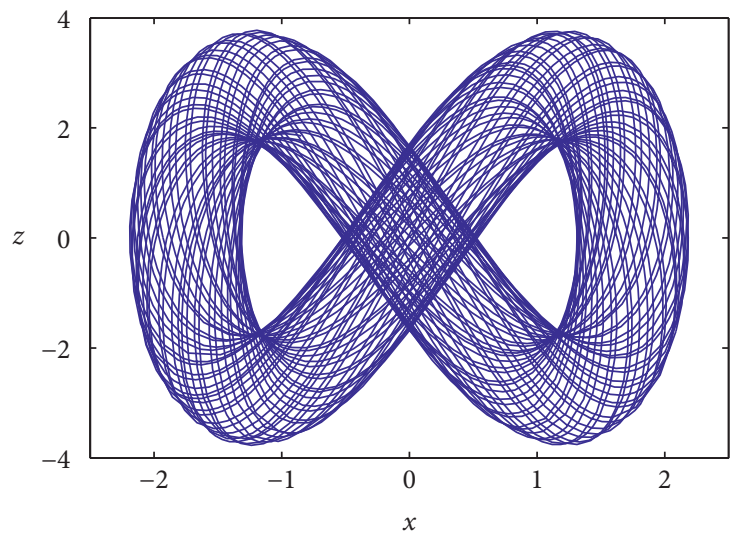

(a)

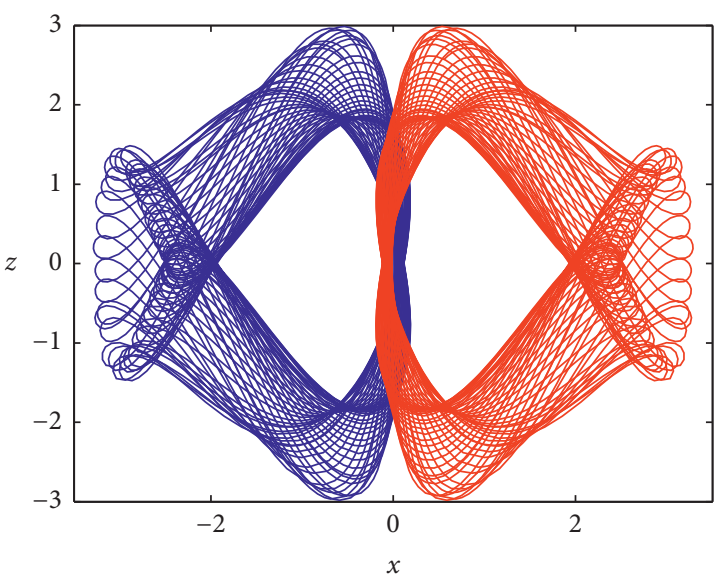

(c)

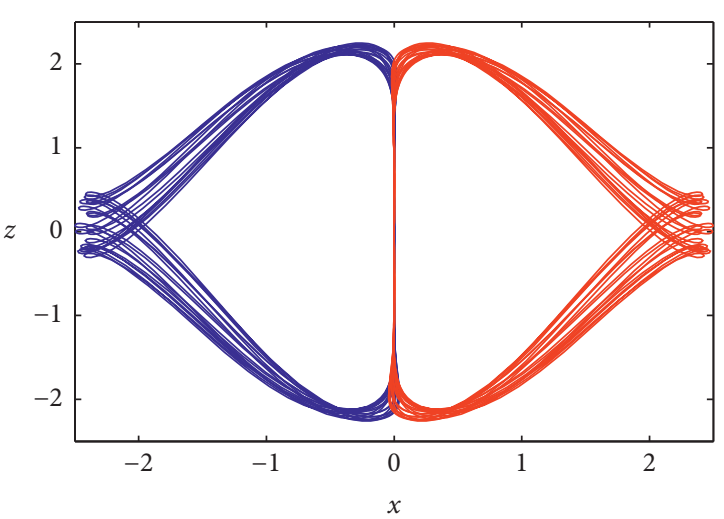

(e)

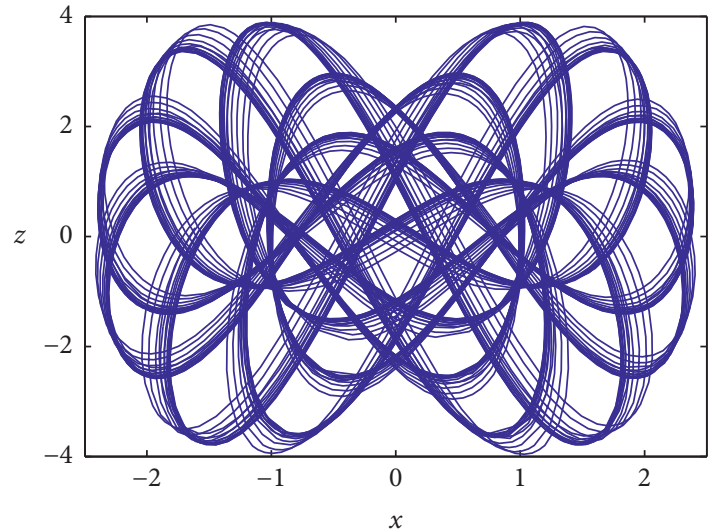

(b)

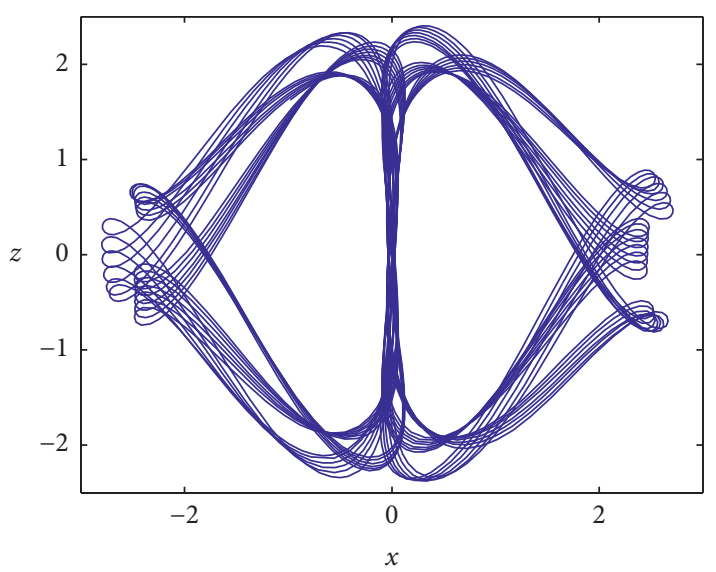

(d)

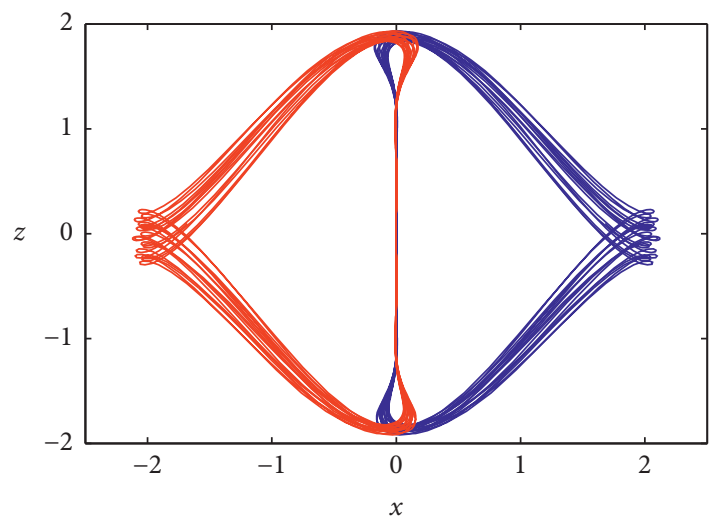

(f)

Figure 2: Continued. 


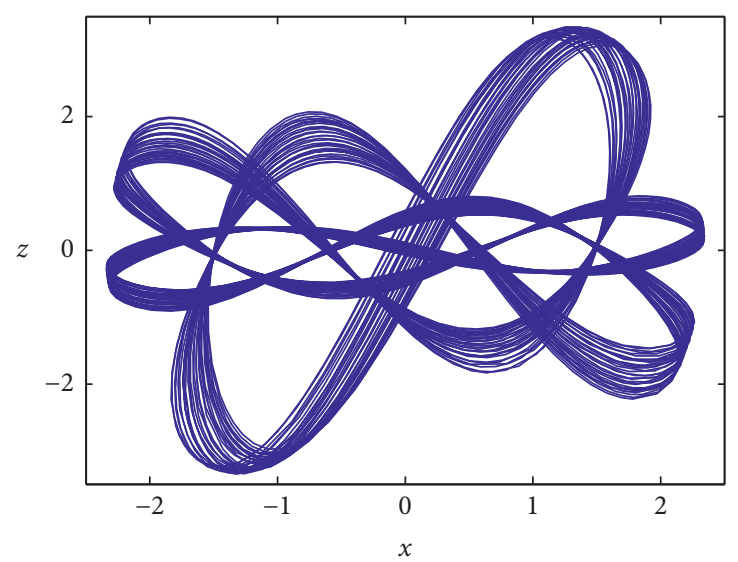

(g)

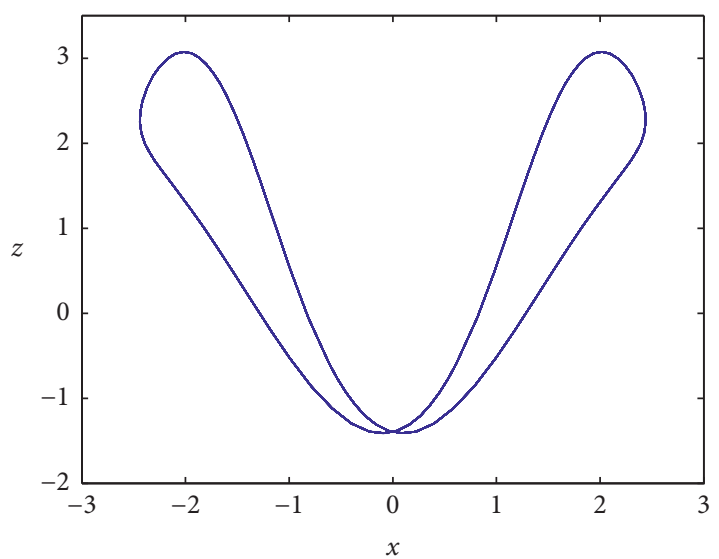

(h)

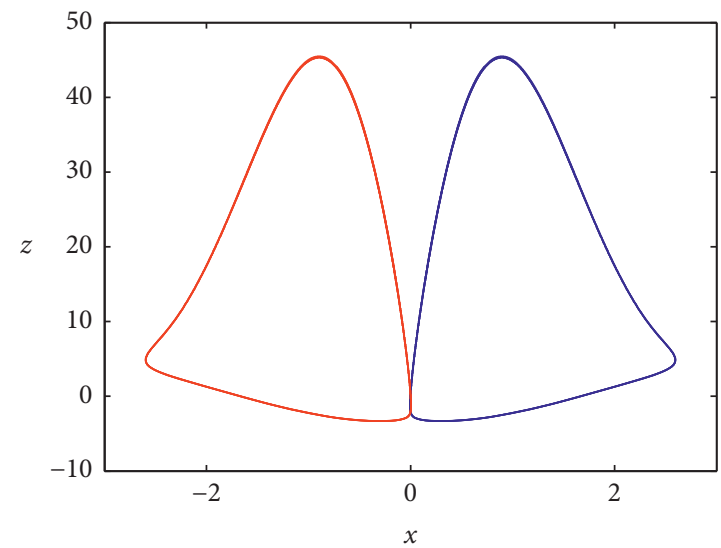

(i)

FIGURE 2: Chaotic flows from system (1) at various values of $a$ and $b$. (a) $a=-5, b=0$. (b) $a=-4, b=0$. (c) $a=-0.8, b=0$. (d) $a=-0.5, b=0$. (e) $a=-0.2, b=0$. (f) $a=-0.15, b=0$. (g) $a=-6.17, b=-1$. (h) $a=-4.5, b=-1$. (i) $a=-0.8, b=-1$.

Figure 3 shows some coexisting chaotic flows of system (1) for some special $a$. From this figure, we can see that a chaotic flow coexists with a limit cycle (Figure 3(b)), two limit cycles coexist (Figure 3(c)), and three limit cycles (a pair of symmetric limit cycles) coexist (Figure 3(d)). The results show that in phase space, there are independent chaotic and quasiperiodic attractive basins, i.e., chaotic flows coexist with periodic limit cycle. Moreover, we plot the basin attractors as shown in Figure 4. The blue area represents the stable domain of system parameters, and the red area represents the unstable domain of system parameters.

\section{Design of Bifurcation-Based Partial-State Feedback Controller}

3.1. Partial-State Feedback Controller. In this section, we will consider system (1) subjected to linear feedback control with desired periodic motions. As far as we know, the simplest way to get a period orbit is through Hopf bifurcation. By using the projection method for the calculation of the first and second Lyapunov coefficients associated to the Hopf bifurcation, the generic and degenerate Hopf bifurcation in the controlled system are studied. Consider the controlled system

$$
\begin{aligned}
& \dot{x}=y, \\
& \dot{y}=-x-y z, \\
& \dot{z}=a-b x^{2}+c y^{2}+e z .
\end{aligned}
$$

When $e \neq 0$, system (6) has only one equilibrium $(0,0,-(a / e))$. Let $\bar{x}=x, \bar{y}=y, \bar{z}=z+(a / e)$, then system (6) will become

$$
\begin{aligned}
& \dot{\bar{x}}=\bar{y}, \\
& \dot{\bar{y}}=-\bar{x}-\bar{y}\left(\bar{z}-\frac{a}{e}\right), \\
& \dot{\bar{z}}=-b \bar{x}^{2}+c \bar{y}^{2}+e \bar{z},
\end{aligned}
$$

and the equilibrium will become an origin $O$; the Jacobian matrix of system (7) at $O$ is

$$
\left(\begin{array}{ccc}
0 & 1 & 0 \\
-1 & \frac{a}{e} & 0 \\
0 & 0 & e
\end{array}\right)
$$




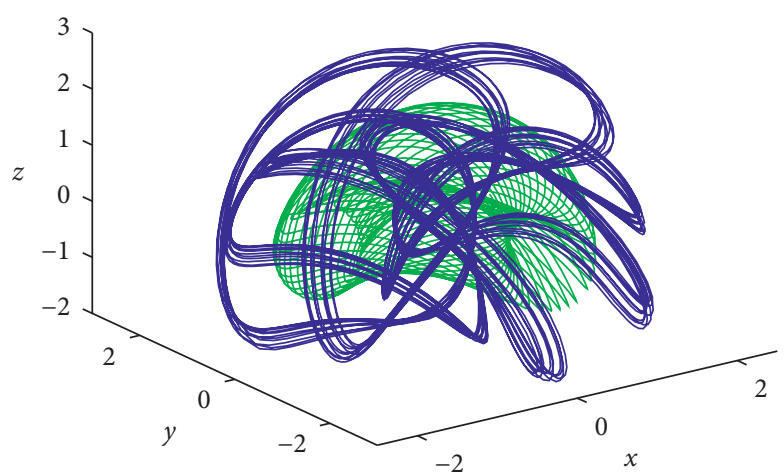

(a)

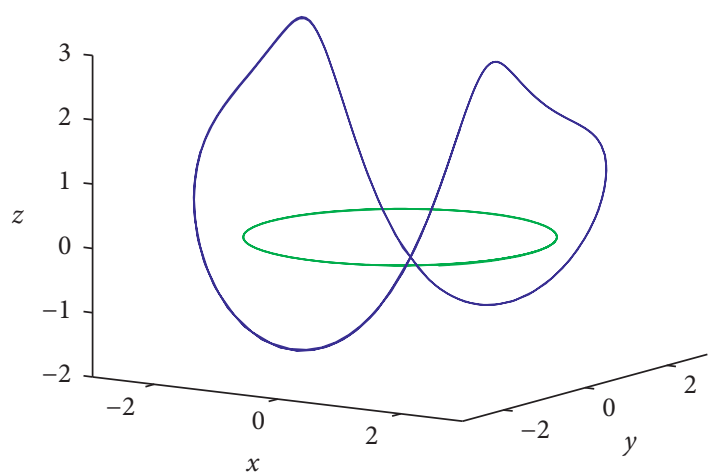

(c)

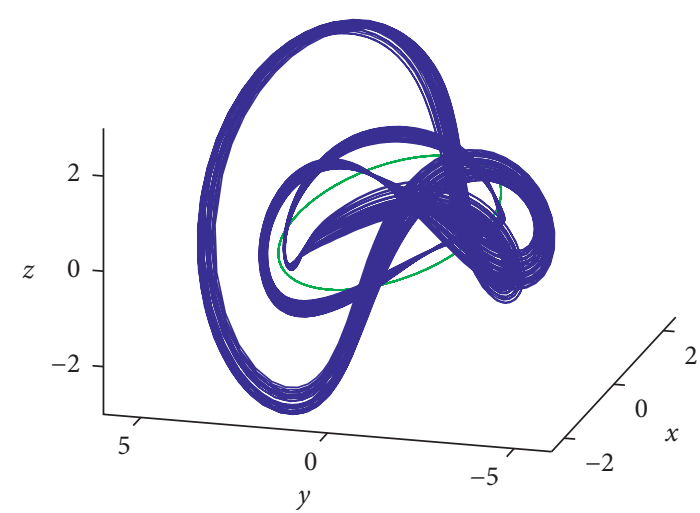

(b)

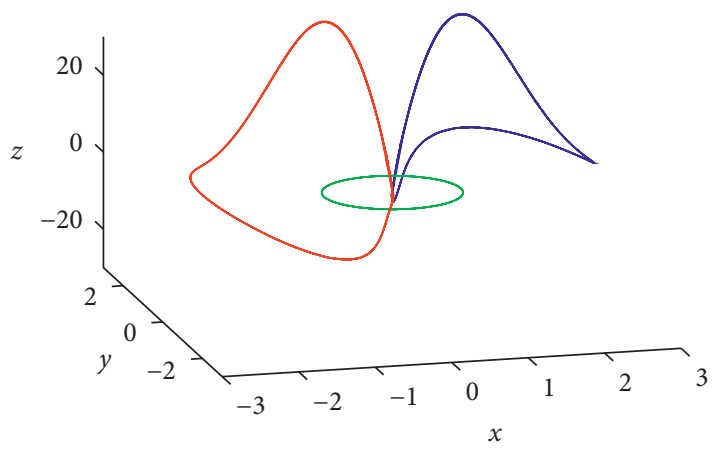

(d)

FIgURe 3: Coexisting chaotic flow of system (1) for $c=1$ at various values of $a$. (a) Chaotic flow coexists a torus for system (1) with $a=-1$, $b=0$. (b) Chaotic flow coexists limit cycle for system (1) with $a=-6.17, b=-1$. (c) Two limit cycles coexisting for system (1) with $a=-4.5$, $b=-1$. (d) Three limit cycles coexisting for system (1) with $a=-0.8, b=-1$.

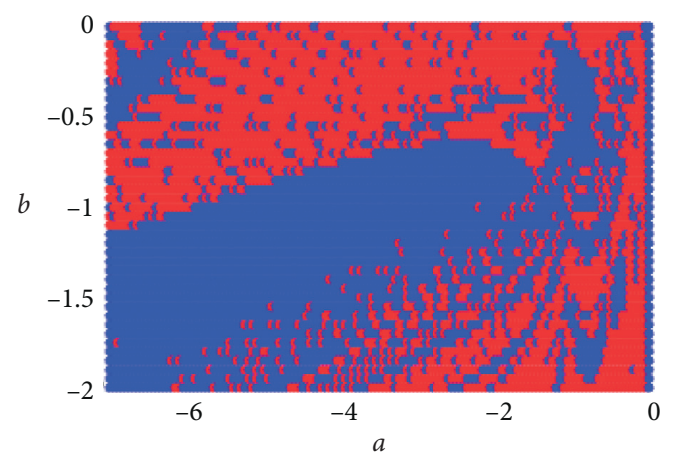

FIgURE 4: Basin attractors of system (1) for $c=1$ at various values of $a$ and $b$.

Meanwhile, the corresponding characteristic equation is

$$
\lambda^{3}-\left(e+\frac{a}{e}\right) \lambda^{2}+(a+1) \lambda-e=0,
$$

and the eigenvalues are

$$
\begin{aligned}
\lambda_{1} & =e, \\
\lambda_{2,3} & =\frac{a \pm \sqrt{a^{2}-4 e^{2}}}{2 e} .
\end{aligned}
$$

Obviously, when $a=a_{0} \stackrel{\text { def }}{=} 0$, system (9) has a pair of pure imaginary roots $\pm i$, and the corresponding eigenvalues are

$$
\begin{aligned}
\lambda_{1} & =e, \\
\lambda_{2,3} & = \pm i .
\end{aligned}
$$

Taking $a$ as the Hopf bifurcation parameter, according to equation (9), we have

$$
\frac{\mathrm{d} \lambda}{\mathrm{d} a}=\frac{(1 / e) \lambda^{2}-1}{3 \lambda^{2}-2(e+(a / e)) \lambda+(a+1)}
$$

Hence,

$$
\begin{aligned}
& \left.\operatorname{Re} \frac{\mathrm{d} \lambda}{\mathrm{d} a}\right|_{a=0, \lambda=i}=\frac{1+e}{2 e\left(1+e^{2}\right)}, \\
& \left.\operatorname{Im} \frac{\mathrm{d} \lambda}{\mathrm{d} a}\right|_{a=0, \lambda=i}=-\frac{1+e}{2\left(1+e^{2}\right)} .
\end{aligned}
$$

When $e<0, e \neq-1$, the transversally condition $\left.\operatorname{Re}(\mathrm{d} \lambda / \mathrm{d} a)\right|_{a=0, \lambda=i} \neq 0$ is satisfied.

Proposition 1. Define the Hopf surface

$$
\mathcal{S}_{h}=\left\{(a, b, c, e) \mid a=a_{0}, e<0, e \neq-1\right\} .
$$


If $(a, b, c, e) \in \mathcal{S}_{h}$, then the Jacobian matrix of (7) at $O$ has one negative real eigenvalue $\lambda_{1}=e$ and a pair of purely imaginary eigenvalues $\lambda_{2,3}= \pm i$.

Meanwhile, we have the following proposition.

Proposition 2. If $(a, b, c, e) \in \mathcal{S}_{h}$, the Hopf bifurcation is at the origin $O$ when a varies and passes through the critical value $a_{0}$.
The following of this section is a review of the projection method for the degenerate Hopf bifurcation.

Consider the differential equation

$$
\dot{X}=f(X, \mu),
$$

where $X \in \mathbb{R}^{3}$ and $\mu \in \mathbb{R}^{4}$. Suppose system (15) has a zero equilibrium when $\mu=\mu_{0}$ and let where

$$
\begin{aligned}
A & =f_{X}\left(X, \mu_{0}\right), \\
B_{i}(X, Y) & =\left.\sum_{j, k=1}^{3} \frac{\partial^{2} F_{i}(\xi)}{\partial \xi_{j} \partial \xi_{k}}\right|_{\xi=0} X_{j} Y_{k}, \\
C_{i}(X, Y, Z) & =\left.\sum_{j, k=1}^{3} \frac{\partial^{3} F_{i}(\xi)}{\partial \xi_{j} \partial \xi_{k} \partial \xi_{l}}\right|_{\xi=0} X_{j} Y_{k} Z_{l},
\end{aligned}
$$

for $i=1,2,3$ and so on for $D, E, K, L$.

Suppose $A$ has a pair of purely imaginary eigenvalues $\lambda_{2,3}= \pm \omega_{0} i, \omega_{0}>0$, let $T^{c}$ be the generalized eigenspace of $A$ corresponding to $\lambda_{2,3}$ and $p, q \in \mathbb{R}^{3}$ be vectors, such that

$$
A q=i \omega_{0} q, A^{T} p=-i \omega_{0} p,\langle p, q\rangle=\sum_{i=1}^{3} \bar{p}_{i} q_{i}=1,
$$

where $A^{T}$ is the transpond of $A$.

Any vector $y \in T^{c}$ can be represented as

$$
y=w q+\overline{w q},
$$

where $w=\langle p, y\rangle \in \mathbb{C}$.

The $2 \mathrm{D}$ center manifold associated to the eigenvalues $\lambda_{2,3}= \pm \omega_{0} i$ can be parameterized by the variables $w$ and $\bar{w}$ by means of an immersion of the form

$$
X=H(w, \bar{w}),
$$

where $H: \mathbb{C}^{2} \longrightarrow \mathbb{R}^{2}$ has a Taylor expansion of the form

$$
H(w, \bar{w})=w q+\overline{w q}+\sum_{2 \leq j+k \leq 7} \frac{1}{j ! k !} h_{j k} w^{j} \bar{w}^{k}+O\left(|w|^{8}\right),
$$

with $h_{j k} \in \mathbb{C}$ and $h_{j k}=\bar{h}_{k j}$. Substituting this expression into (16), we have

$$
H_{w} \dot{w}+H_{\bar{w}} \dot{\bar{w}}=F(H(w, \bar{w})) .
$$

The complex vectors $h_{j k}$ are obtained solving system (22). Taking into account the coefficients of $F$, system (22) can be written as

$$
\dot{w}=i \omega_{0} w+\frac{1}{2} G_{21} w|w|^{2}+\frac{1}{12} G_{32} w|w|^{4}+\frac{1}{144} G_{43} w|w|^{6}+O\left(|w|^{8}\right) .
$$

The first Lyapunov coefficient $l_{1}$ is defined by

$$
l_{1}=\frac{1}{2} \operatorname{Re}\left(G_{21}\right)
$$

where $G_{21}=\left\langle p, \mathscr{H}_{21}\right\rangle$, and $\mathscr{H}_{21}=C(q, q, \bar{q})+B\left(\bar{q}, h_{20}\right)$ $-2 B\left(q, h_{11}\right)$.

Define $\mathscr{H}_{32}$ as

$$
\begin{aligned}
\mathscr{H}_{32}= & 6 B\left(h_{11}, h_{21}\right)+B\left(\bar{h}_{20}, h_{30}\right)+3 B\left(\bar{h}_{12}, h_{20}\right)+3 B\left(q, h_{22}\right)+2 B\left(\bar{q}, h_{31}\right)+6 C\left(q, h_{11}, h_{11}\right)+3 C\left(q, \bar{h}_{20}, h_{20}\right) \\
& +3 C\left(q, q, \bar{h}_{21}\right)+6 C\left(q, \bar{q}, h_{21}\right)+6 C\left(\bar{q}, h_{20}, h_{11}\right)+C\left(\bar{q}, \bar{q}, h_{30}\right)+D\left(q, q, q, \bar{h}_{20}\right) \\
& +6 D\left(q, q, \bar{q}, h_{11}\right)+3 D\left(q, \bar{q}, \bar{q}, h_{20}\right)+E(q, q, q, \bar{q}, \bar{q})-6 G_{21} h_{21}-3 \bar{G}_{21} h_{21} .
\end{aligned}
$$

The second Lyapunov coefficient $l_{2}$ is defined by

$$
l_{2}=\frac{1}{12} \operatorname{Re}\left(G_{32}\right),
$$


where $G_{21}=\left\langle p, \mathscr{H}_{32}\right\rangle$.

The third Lyapunov coefficient $l_{3}$ is defined by

$$
l_{3}=\frac{1}{144} \operatorname{Re}\left(G_{43}\right)
$$

where $G_{43}=\left\langle p, \mathscr{H}_{43}\right\rangle$. The expression for $\mathscr{H}_{43}$ is too large to be put in print and can be found in [47].

3.2. Hopf Bifurcation Analysis. In this section, we study the stability of $O$ for parameters in $\delta_{h}$. Using the notation of the previous section, system (7) can be written as

$$
\dot{X}=A X+\frac{1}{2} B(X, X)
$$

where

$$
\begin{aligned}
& A=\left(\begin{array}{ccc}
0 & 1 & 0 \\
-1 & 0 & 0 \\
0 & 0 & e
\end{array}\right), \\
& X=\left(\begin{array}{l}
\bar{x} \\
\bar{y} \\
\bar{z}
\end{array}\right),
\end{aligned}
$$

and the multilinear symmetric function

$$
B(X, Y)=\left(\begin{array}{c}
0 \\
-X_{2} Y_{3}-X_{3} Y_{2} \\
-2 b X_{1} Y_{1}+2 c X_{2} Y_{2}
\end{array}\right) .
$$

Meanwhile, we can calculate $q, p$ such that $\langle p, q\rangle=1$ and

$$
\begin{aligned}
& q=\left(\begin{array}{lll}
-i & 1 & 0
\end{array}\right)^{T}, \\
& p=\left(\begin{array}{lll}
-\frac{1}{2} i & \frac{1}{2} & 0
\end{array}\right)^{T}
\end{aligned}
$$

The complex vectors $h_{11}, h_{20}$, and $h_{30}$ are

$$
\begin{aligned}
& h_{11}=-A^{-1} B(q, \bar{q})=\left(\begin{array}{lll}
0 & 0 & \frac{2(b-c)}{e}
\end{array}\right)^{T}, \\
& h_{20}=\left(2 i \omega_{0} I-A\right)^{-1} B(q, q)=\left(\begin{array}{lll}
0 & 0 & \frac{2(b+c)}{2 i-e}
\end{array}\right)^{T}, \\
& h_{30}=3\left(3 i \omega_{0} I-A\right)^{-1} B\left(q, h_{20}\right)=\left(\frac{3(b+c)}{4(2 i-e)} \frac{9(b+c) i}{4(2 i-e)} 0\right)^{T} \text {. }
\end{aligned}
$$

The complex number $G_{21}$ has the form

$$
G_{21}=-\frac{2(b-c)}{e}-\frac{b+c}{2 i-e} \text {. }
$$

Defining $b=b_{0} \triangleq\left(\left(3 e^{2}+8\right) c /\left(e^{2}+8\right)\right)$ and the following subsets of the Hopf surface $\mathcal{S}_{h}$,

$$
\begin{aligned}
& \mathcal{U}=\left\{(a, b, c, e) \mid a=a_{0}, b>\frac{3 e^{2}+8}{e^{2}+8} c, e<0, e \neq-1\right\}, \\
& \mathcal{S}=\left\{(a, b, c, e) \mid a=a_{0}, b<\frac{3 e^{2}+8}{e^{2}+8} c, e<0, e \neq-1\right\} .
\end{aligned}
$$

Hence, we have the following theorem.

Theorem 1. The first Lyapunov coefficient at $O$ for $p a-$ rameters in $\mathcal{S}_{h}$ is given by

$$
l_{1}=\frac{\left(3 e^{2}+8\right) c-\left(e^{2}+8\right) b}{2 e\left(e^{2}+4\right)} .
$$

If $\left(3 e^{2}+8\right) c-\left(e^{2}+8\right) b \neq 0$, system (7) has a transversal Hopf point at $O$ for $(a, b, c, e) \in \mathcal{S}_{h}$. More specifically, if $(a, b, c, e) \in \mathcal{U}$, then the Hopf point at $O$ is unstable (weak repelling focus), and for each $a<a_{0}$, but close to $a_{0}$, there exists an unstable limit cycle near the asymptotically stable equilibrium $O$; if $(a, b, c, e) \in \mathcal{S}$, then the Hopf point at $O$ is stable (weak attractor focus), and for each $a>a_{0}$, but close to $a_{0}$, there exists a stable limit cycle near the unstable equilibrium point $\mathrm{O}$.

If $a=-0.01, b=1, c=0, e=-2$, then $\left(3 e^{2}+8\right) c-$ $\left(e^{2}+8\right) b=-12 \neq 0$, and $b=1>\left(\left(3 e^{2}+8\right) /\left(e^{2}+8\right)\right) c=0$, so $(a, b, c, e) \in \mathcal{U}$. According to Theorem 1, system (7) is unstable, and for each $a<a_{0}$, but close to $a_{0}$, there exists an unstable limit cycle near the asymptotically stable equilibrium $O$. We can get the numerical simulation results as shown in Figure 5(a); there exists an asymptotically stable equilibrium $O$.

If $a=0.01, b=-1, c=1, e=-2$, then $\left(3 e^{2}+8\right) c-$ $\left(e^{2}+8\right) b=32 \neq 0$, and $b=-1<\left(\left(3 e^{2}+8\right) /\left(e^{2}+8\right)\right) c=(20 /$ 12), so $(a, b, c, e) \in \mathcal{S}$. According to Theorem 1 , system (7) is stable, and for each $a>a_{0}$, but close to $a_{0}$, there exists a stable limit cycle near the unstable equilibrium point $O$. We can get the numerical simulation results as shown Figure 5(b), and there exists an unstable equilibrium $O$.

The first Lyapunov coefficient vanishes on the straight line:

$$
\mathcal{S}_{l}=\left\{(a, b, c, e) \mid a=a_{0}, b=\frac{3 e^{2}+8}{e^{2}+8} c, e<0, e \neq-1\right\} .
$$

The second Lyapunov coefficient on the straight line $\delta_{l}$ where the first Lyapunov coefficient vanishes will be calculated in the following.

The complex vector $h_{21}$ can be solved by solving the following equation:

$$
\left(\begin{array}{cc}
i I-A & q \\
\bar{p}^{T} & 0
\end{array}\right)\left(\begin{array}{c}
h_{21} \\
s
\end{array}\right)=\left(\begin{array}{c}
B\left(\bar{q}, h_{20}\right)+2 B\left(q, h_{11}\right)-G_{21} q \\
0
\end{array}\right)
$$




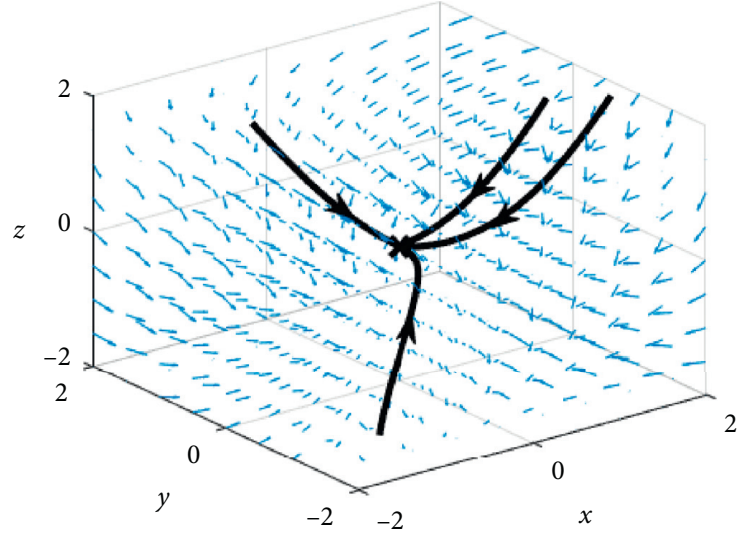

(a)

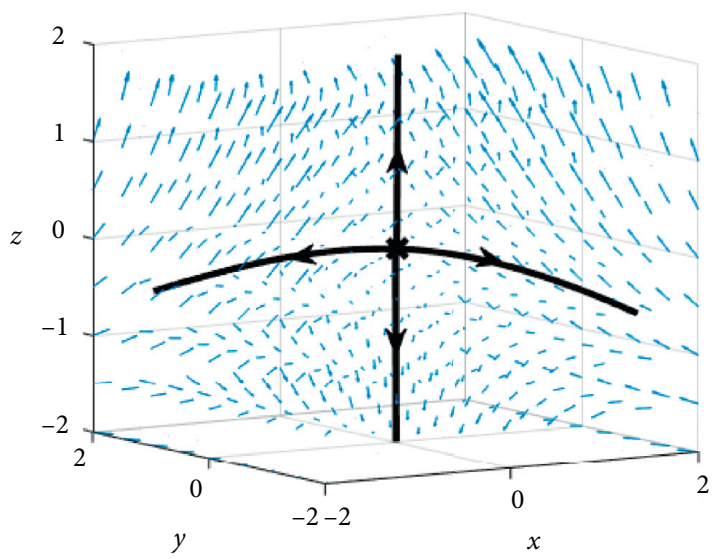

(b)

Figure 5: Stability of control parameters under the first Lyapunov exponent. (a) $a=-0.01, b=1, c=0, e=-2$. (b) $a=0.01, b=-1, c=1, e=-2$.

and we have

$$
h_{21}=\left(-\frac{4 c(e i+2)}{\left(e^{2}+8\right)(2 i-e)} \frac{4 c(e i+2) i}{\left(e^{2}+8\right)(2 i-e)} 0\right)^{T} \text {. }
$$

From the coefficients of the term $w^{4}, w^{3} \bar{w}$, and $w^{2} \bar{w}^{2}$ in (22), we have

$$
\begin{aligned}
& h_{40}=(4 i I-A)^{-1}\left[3 B\left(h_{20}, h_{20}\right)+4 B\left(q, h_{30}\right)\right] \\
& =\left(\begin{array}{lll}
0 & 0 & \frac{48 c^{2}\left(e^{2}+4\right)\left(3 e^{2}+16\right) i}{(4 i-e)\left(e^{2}+8\right)^{2}(2 i-e)}
\end{array}\right)^{T} \\
& h_{31}=(2 i I-A)^{-1}\left[3 B\left(q, h_{21}\right)+B\left(\bar{q}, h_{30}\right)+3 B\left(h_{20}, h_{11}\right)-3 G_{21} h_{20}\right] \\
& =\left(\begin{array}{lll}
0 & 0 & \frac{48 c^{2}\left(16 e^{3} i-e^{4}+8 e i+8 e^{2}+16\right)}{\left(e^{2}+8\right)^{2}(2 i-e)^{3}}
\end{array}\right)^{T} \\
& h_{22}=-A^{-1}\left[2 B\left(h_{11}, h_{11}\right)+2 B\left(q, \bar{h}_{21}\right)+2 B\left(\bar{q}, h_{21}\right)+B\left(\bar{h}_{20}, h_{20}\right)\right] \\
& =\left(00-\frac{64\left(u e^{6}+20 u e^{4}+c^{2} e^{2}+128 e^{2} u+4 c^{2}+256 u\right)}{e\left(e^{2}+8\right)^{2}}\right)^{T}, \\
& G_{32}=\frac{12}{\left(e^{2}+8\right)^{2}(2 i-e)^{3}(e i+2) e}\left(\begin{array}{r}
-512 c^{2}-32768 u i-3 v e^{13}-88 u e^{9}+24 i v e^{12}+480 i v e^{10} \\
-12 i u e^{10}+2688 i v e^{8}-16 i c^{2} e^{4}-1536 i v e^{6}-49152 i v e^{4} \\
-9830 i v e^{2}+3136 i u e^{6}+25088 i u e^{4}+576 i c^{2} e^{2}+45056 i u e^{2} \\
-49152 v e+73728 u e+11520 v e^{7}+1056 v e^{9}-1472 u e^{7} \\
+42240 v e^{5}-5504 u e^{5}+36864 v e^{3}+14336 u e^{3}-160 c^{2} e^{3}+896 c^{2} e
\end{array}\right),
\end{aligned}
$$


where

$$
\begin{aligned}
& u=\left|\frac{c^{2}(e i+2)^{2}}{\left(e^{2}+8\right)^{2}(2 i-e)^{2}}\right| \\
& v=\left|\frac{c^{2}}{\left(e^{2}+8\right)^{2}(2 i-e)^{2}}\right|
\end{aligned}
$$

Hence, we have the following theorem.

Theorem 2. The second Lyapunov coefficient at $O$ for parameter in $\mathcal{S}_{l}$ is given by

$$
\left.l_{2}\right|_{\mathcal{S}_{l}}=\frac{4 c^{2}\left(3 e^{6}+44 e^{4}+192 e^{2}+256+4\left(e^{2}+8\right)^{2}\right)}{e\left(e^{2}+4\right)\left(e^{2}+8\right)^{3}}<0 .
$$

Moreover, the Hopf point at $\mathrm{O}$ is stable (weak attractor focus), and for each $a>a_{0}$, but close to $a_{0}$, there exists a stable limit cycle near the unstable equilibrium point $O$.

If $a=0.01, b=5, c=3, e=-2$, then $b=\left(\left(3 e^{2}+8\right) /\right.$ $\left.\left(e^{2}+8\right)\right) c=5$, and $(a, b, c, e) \in S_{l}$. According to Theorem 2 , the Hopf point at $O$ is unstable. We can get the numerical simulation results as shown in Figure 6.

\section{Circuit Model Construction and Simulation}

4.1. Circuit Model Construction. In this section, we adopted the analog circuit to illustrate the correctness of above analyzes. The chaotic system (1) can be rewritten as follows when $b=-1, c=1$ :

$$
\left\{\begin{array}{l}
\dot{x}=y, \\
\dot{y}=-x-y z, \\
\dot{z}=a+x^{2}+y^{2}+e z .
\end{array}\right.
$$

Then, according to system (42), the circuit principle diagram is designed as shown in Figure 7 . The analog circuit is constructed by the AD633 analog multipliers, resistors, LM741 operational amplifiers, and capacitors. The parameters are set as that the gain of multiplier AD633 is 0.1 , the voltage of the operational amplifier LM741 is bounded as $15 \mathrm{~V}$, and the output saturated voltage of the operational amplifier LM741 is bounded as $13.5 \mathrm{~V}$; the capacitors are chosen as $C_{1}=C_{2}=C_{3}=10 \mathrm{nF}$. We let the time constant $\tau=t / 10 R_{i} C_{i}$; then, the corresponding circuit equations can be rewritten as

$$
\left\{\begin{array}{l}
\frac{\mathrm{d} x}{\mathrm{~d} \tau}=-10\left(\frac{R_{12}}{R_{11}} \cdot(-y)\right), \\
\frac{\mathrm{d} y}{\mathrm{~d} \tau}=-10\left(\frac{R_{23}}{R_{21}} \cdot x+\frac{R_{23}}{10 R_{22}} \cdot y z\right), \\
\frac{\mathrm{d} z}{\mathrm{~d} \tau}=-10\left(-\frac{e R_{35}}{100} \cdot(-z)+\frac{R_{35}}{10 R_{32}} \cdot\left(-x^{2}\right)+\frac{R_{35}}{10 R_{33}}\left(-y^{2}\right)+\frac{R_{35}}{R_{34}} \cdot(-a)\right)
\end{array}\right.
$$

In the circuit of the state equation $x$, the values of resistances are $R_{11}=100 \mathrm{k} \Omega, R_{12}=R_{13}=R_{14}=R_{15}=10 \mathrm{k} \Omega$. In the circuit of the state equation $y$, the values of resistances are $R_{21}=100 \mathrm{k} \Omega, R_{22}=R_{23}=R_{24}=R_{25}=R_{26}=10 \mathrm{k} \Omega$. In the circuit of the state equation $z$, the values of resistances are $\quad R_{34}=100 \mathrm{k} \Omega, R_{32}=R_{33}=R_{35}=R_{36}=R_{37}=R_{38}=$ $10 \mathrm{k} \Omega, R_{31}=(100 /(-e)) \mathrm{k} \Omega$, and $a$ can take different values. In order to set the initial value, we add a power supply and two switches at both ends of the capacitor $C_{2}$ and provide the initial capacitance value by charging the capacitor $C_{2}$.

4.2. Circuit Simulation. We consider the following two cases. In the case $\mathrm{A}$, we consider $e=0$, and the dynamic behavior will be reproduced by the simulation results. Then, in the case $\mathrm{B}$, the control term $e z$ will be added to the system, and the simulation results will demonstrate the Hopf bifurcation analysis results are as follows:
Case A $(e=0)$ : in this case, the control term $e z$ is not considered. The simulation results of the digital oscilloscope are shown in Figure 8. From Figure 8, it can be observed different motion states of system (1) for different values of the parameter $a, b$ under $c=1$. From Figures 8(a) and 8(b), the rotationally symmetric chaotic flow can be observed under the parameters $a=$ $5, b=0$ or $a=-4, b=0$. From Figures $8(\mathrm{c})$ and $8(\mathrm{~d})$, under the parameters $a=5, b=0$ or $a=-0.2, b=0$, the symmetric pair of chaotic flows can be observed. From Figure 8(e), under the parameters $a=-4.5$ and $b=-1$, the symmetric limit cycle can be observed. From Figure 8(f), under the parameters $a=-0.8$ and $b=-1$, the symmetric pair of limit cycles can observed. Thus, the results of analog circuit verify the results of the numerical simulation as shown in Figure 2.

From Section 2.2, we know that system (1) has coexistence. In this section, we adopt the circuit simulation 


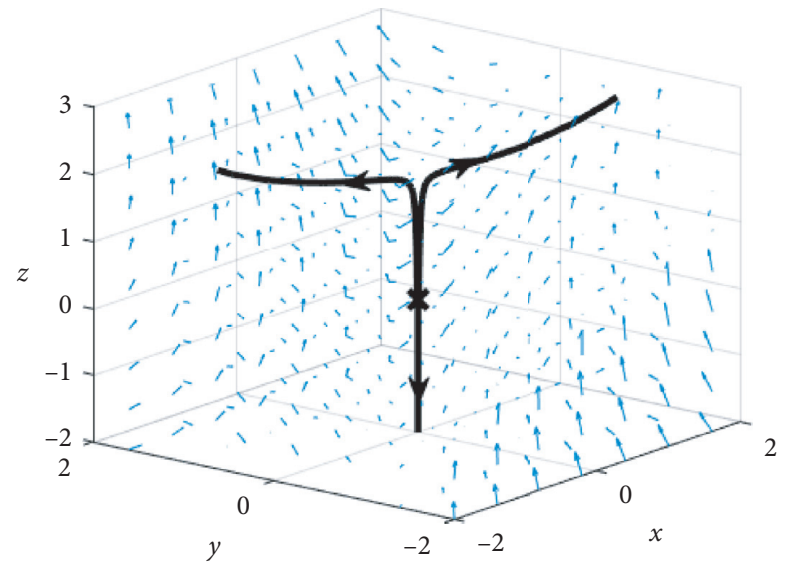

FIGURE 6: Stability of control parameters under the second Lyapunov exponent.

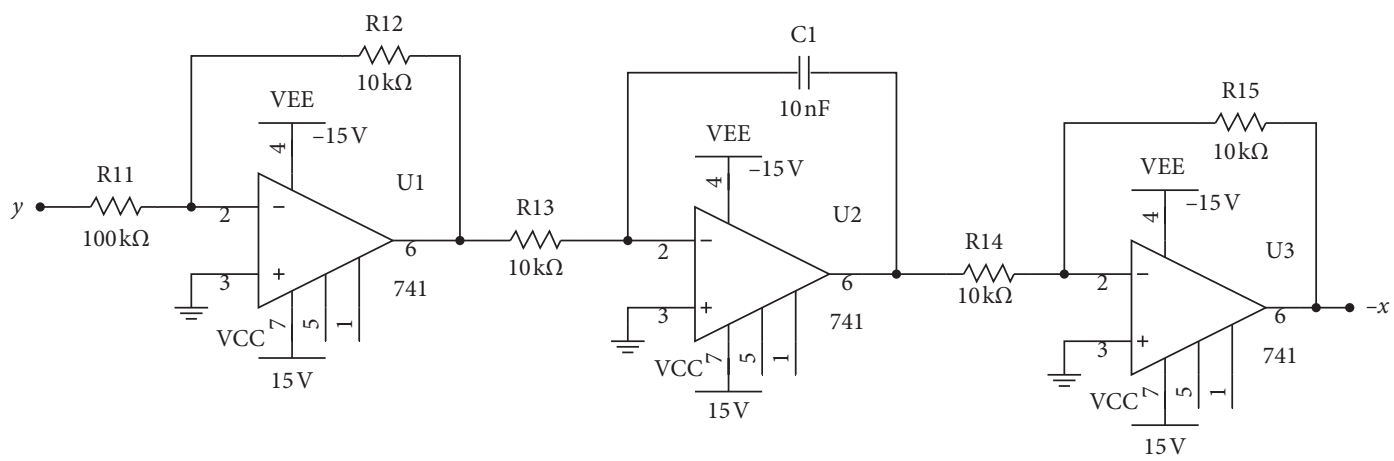

(a)

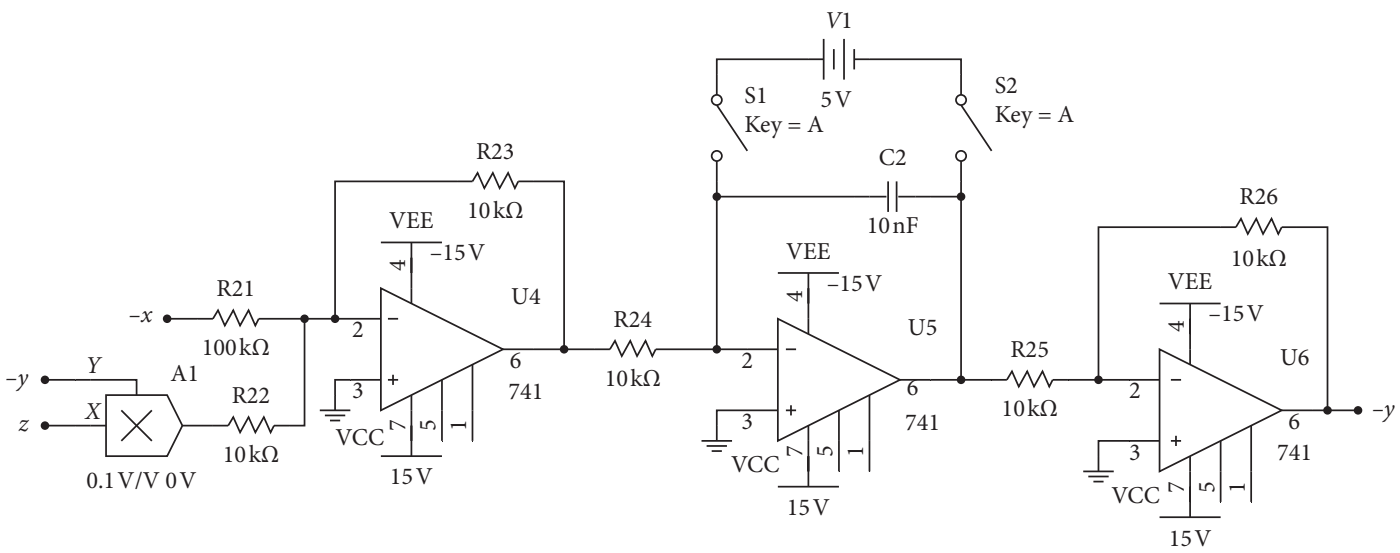

(b)

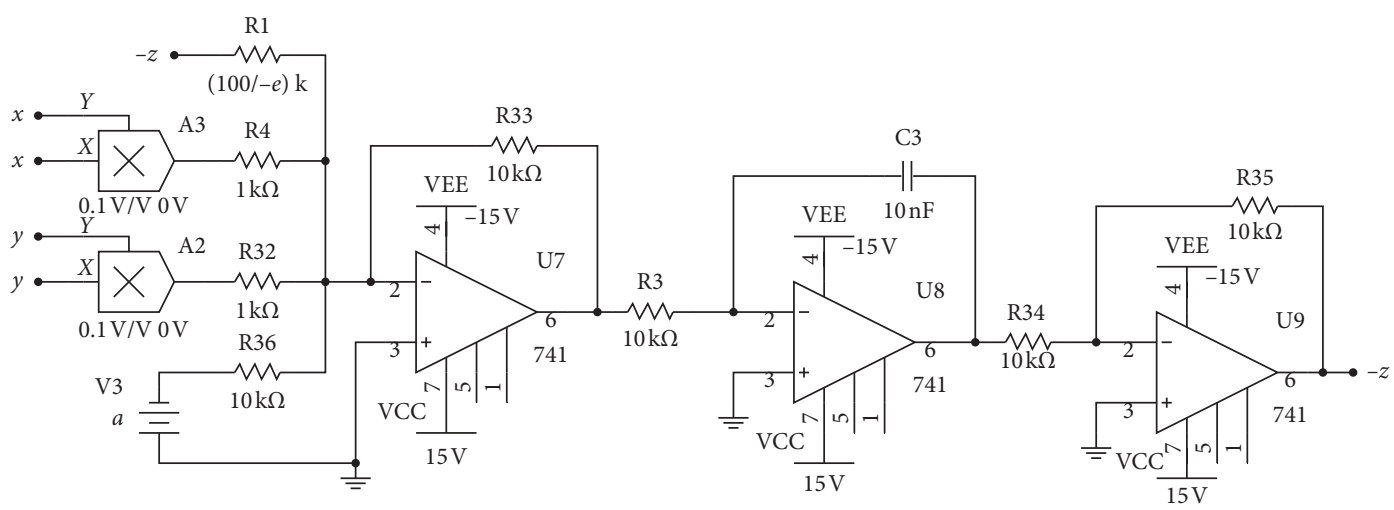

(c)

Figure 7: Circuit principle diagram. (a) Circuit principle diagram of state $x$. (b) Circuit principle diagram of state $y$. (c) Circuit principle diagram of state $z$. 


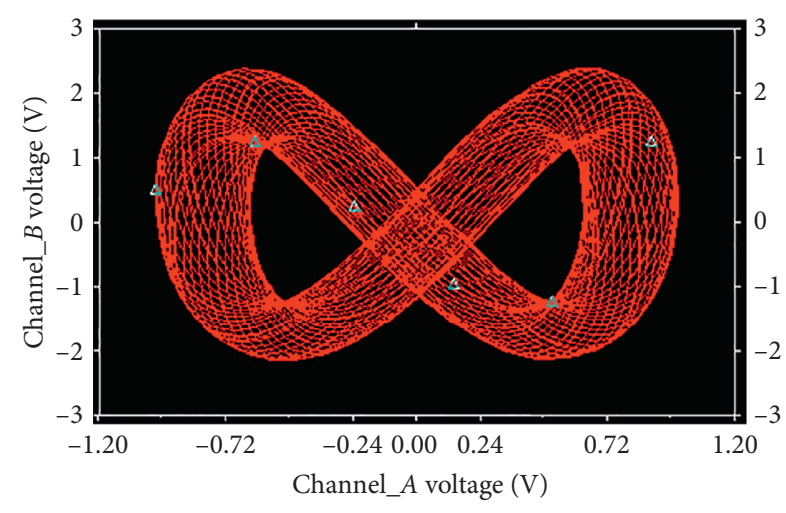

(a)

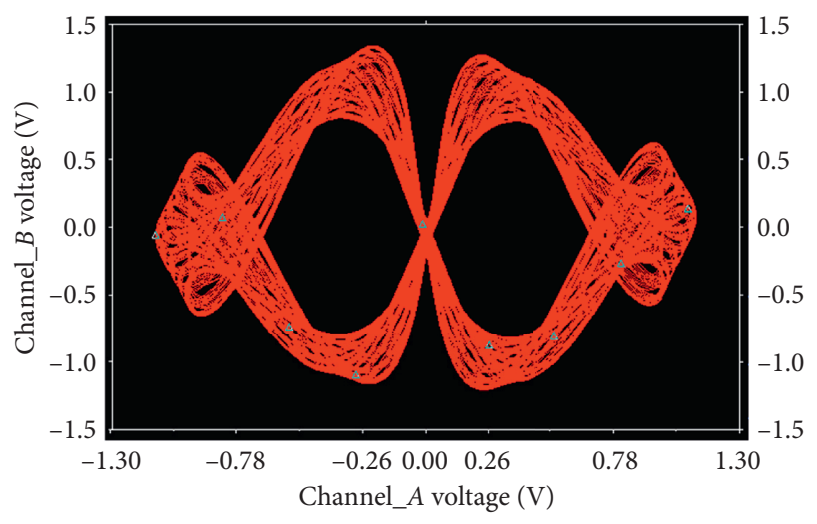

(c)

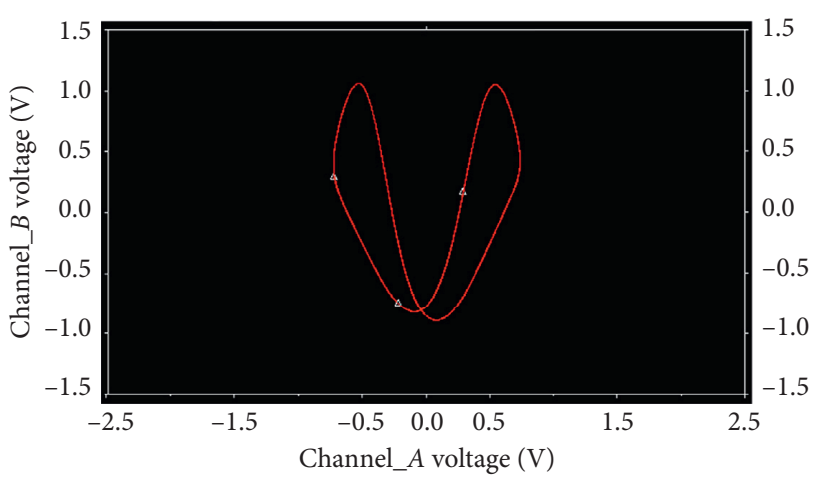

(e)

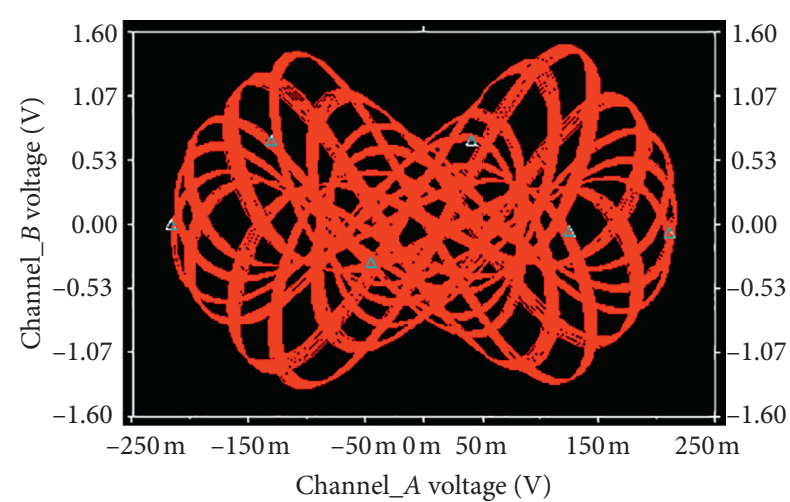

(b)

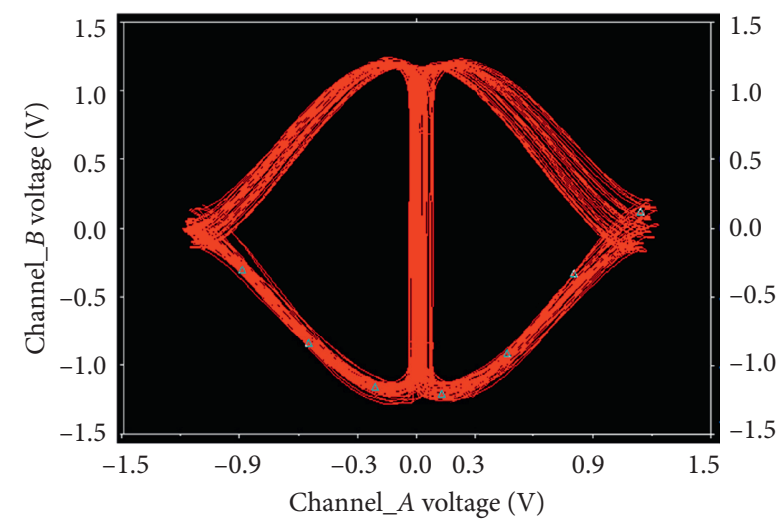

(d)

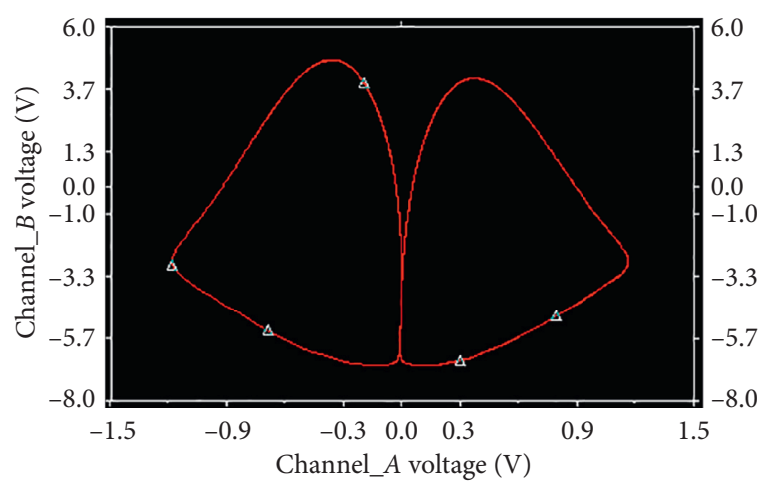

(f)

Figure 8: Circuit simulation results of case A. (a) $a=-5, b=0$. (b) $a=-4, b=0$. (c) $a=-0.8, b=0$. (d) $a=-0.2, b=0$. (e) $a=-4.5, b=-1$. (f) $a=-0.8, b=-1$.

to reproduce the coexistence as shown in Figure 3(c). The system parameters are $a=-4.5, b=-1, c=1$, and the initial values are $(0,5,0)$ and $(0,2.12,0)$; we can get the circuit simulation results as shown in Figure 9. Since Figure $3(\mathrm{c})$ is the $x-y-z$ phase diagram, Figure 9 uses (a)-(d) to reproduce the diagram, where Figure $9(\mathrm{a})$ is the $x-y$ map of the blue limit cycle, Figure 9 (b) is the $x-z$ map of the blue limit cycle, Figure $9(\mathrm{c})$ is the $x-y$ map of the green limit cycle, and Figure $9(\mathrm{~d})$ is the $x-z$ map of the green limit cycle. We can see that the results of analog circuit verify the numerical simulation results as shown in Figure 3(c).
Case B $(e \neq 0)$ : when the control term $e=0$, for $a=$ $-0.2, b=0, c=1$ and $a=0.001, b=0, c=1$, the simulation results are shown in Figures 10(a) and 10(c). From Figures 10(a) and 10(c), the chaotic phenomenon can be observed. Then, the control term $e z$ is added into system, based on the analysis of Section 3, and we let $e=-2$. The simulation results of $e=-2$ are shown in Figures 10(b) and 10(d); obviously, the chaotic phenomenon in Figures 10(a) and 10(c) can be eliminated.

In the simulation experiment, we found an interesting phenomenon. Under the parameter $a=-0.05, b=1, c=0.5$, 


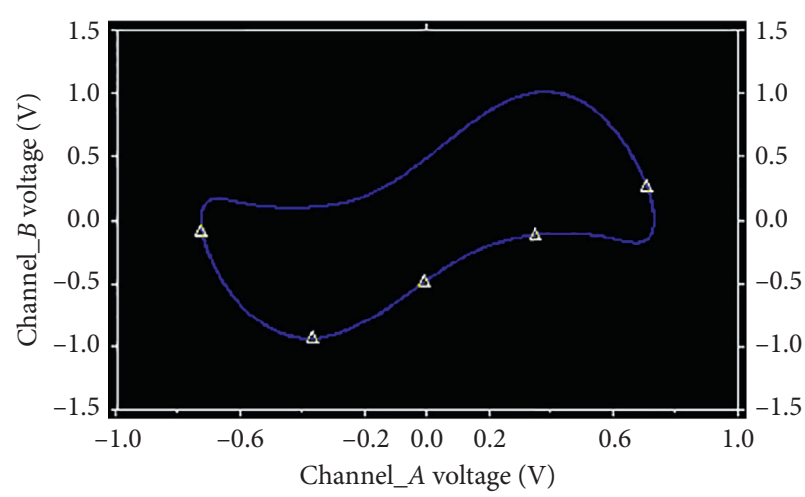

(a)

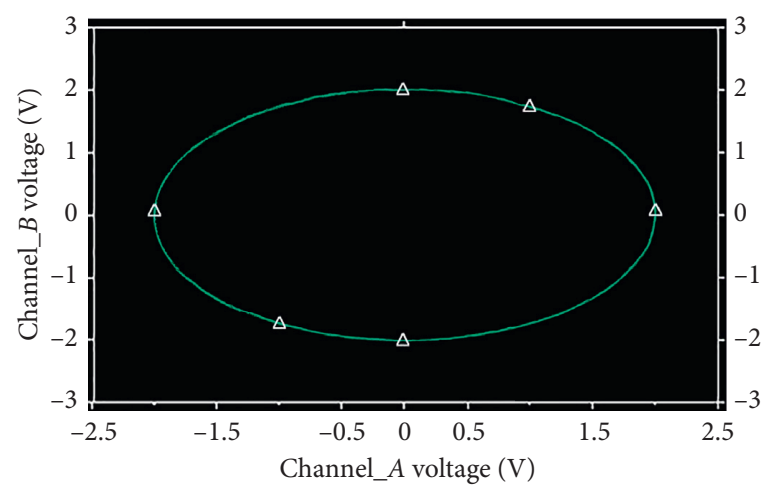

(c)

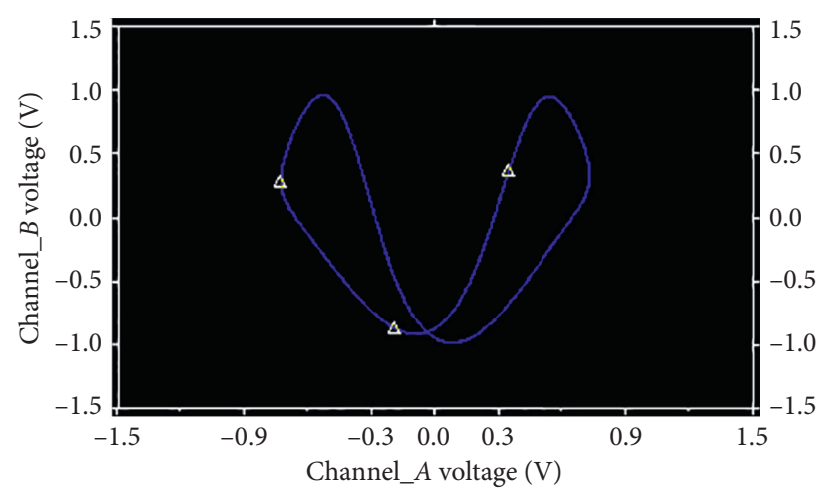

(b)

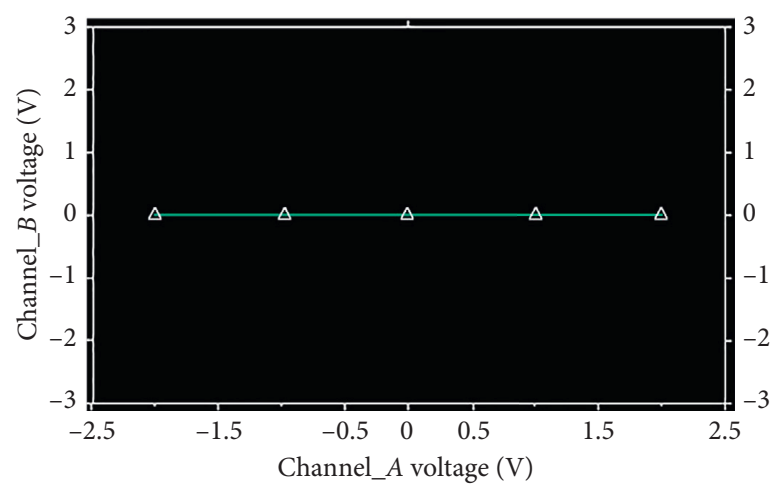

(d)

FIGURE 9: Circuit simulation results of coexisting symmetric chaotic flow for system (1). (a) $x-y$ map. Initial value is $(0,5,0)$. (b) $x-z$ map. Initial value is $(0,5,0)$. (c) $x-y$ map. Initial value is $(0,2.12,0)$. (d) $x-z$ map. Initial value is $(0,2.12,0)$.

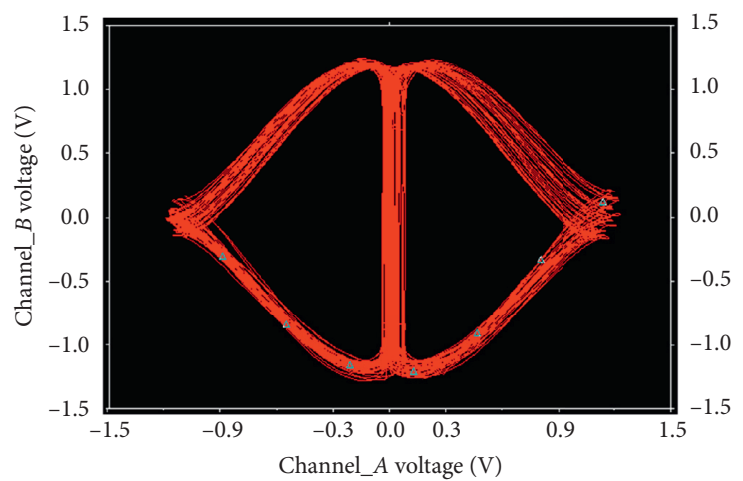

(a)

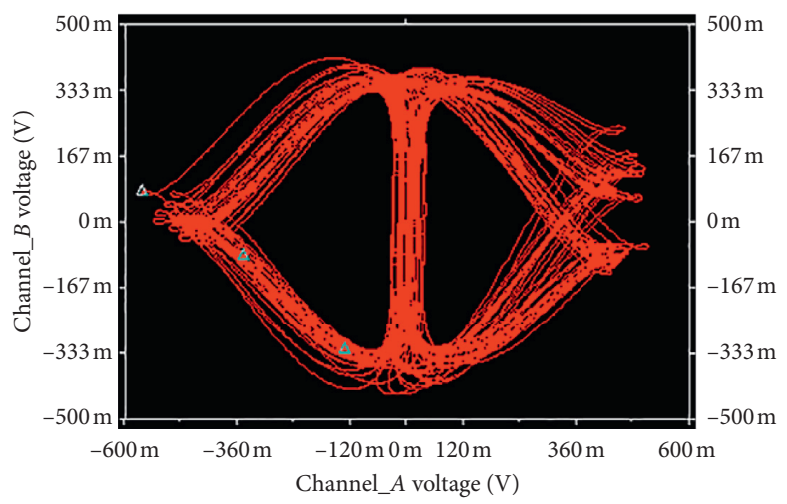

(c)

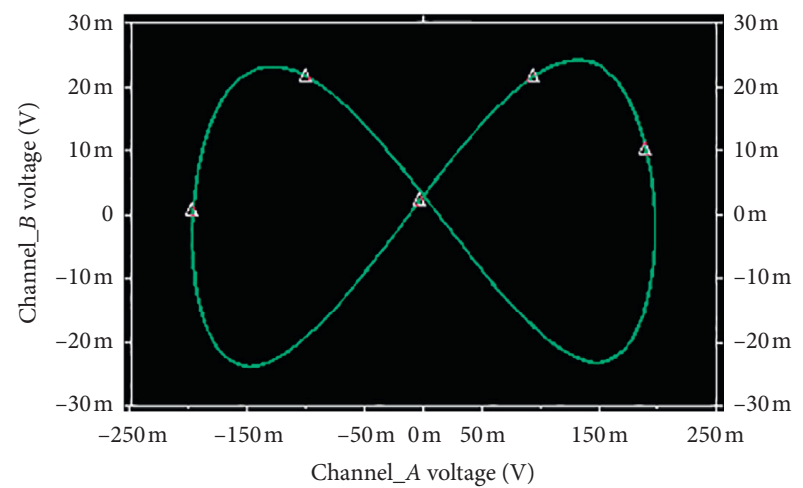

(b)

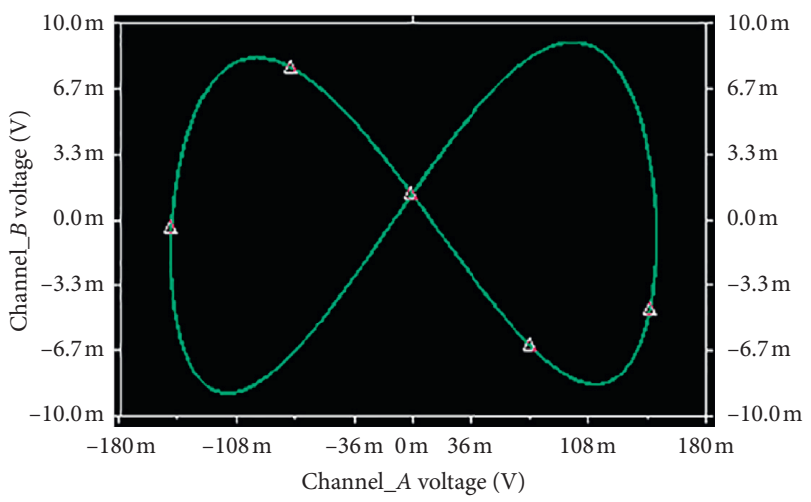

(d)

Figure 10: Circuit simulation results of case B. (a) $a=-0.2, b=0, c=1, e=0$. (b) $a=-0.2, b=0, c=1, e=-2$. (c) $a=0.001, b=0, c=1, e=0$. (d) $a=0.001, b=0, c=1, e=-2$. 


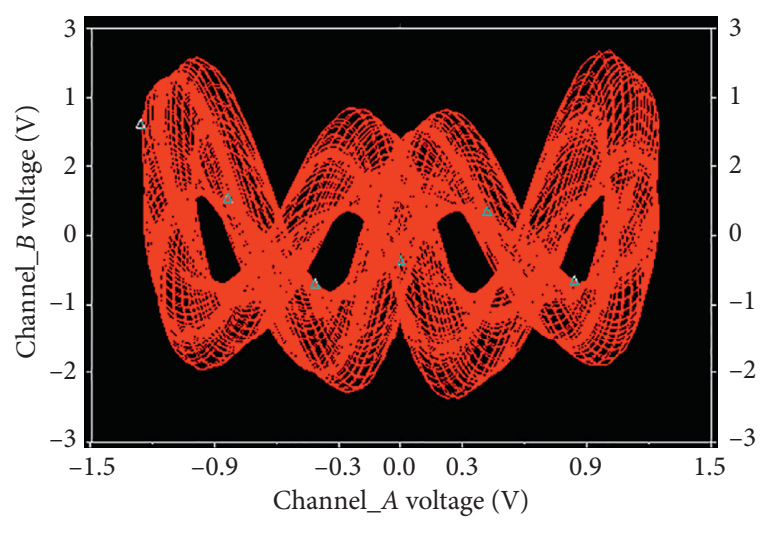

(a)

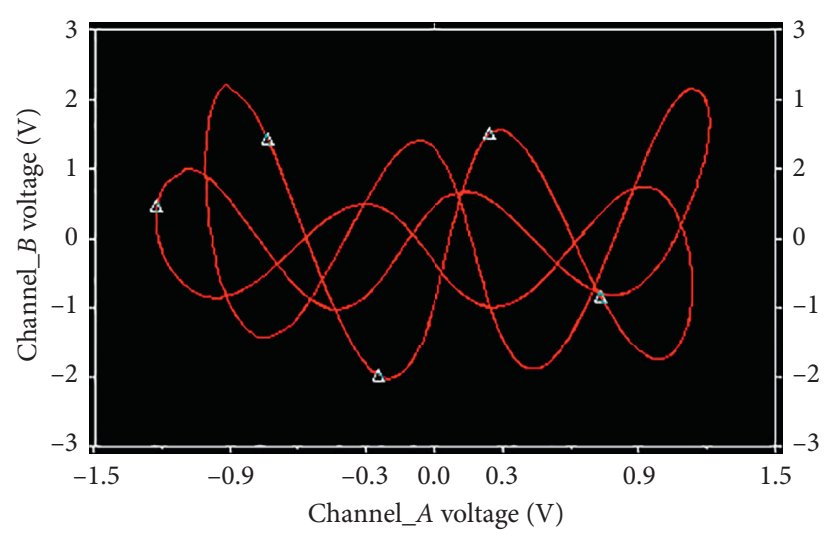

(b)

FIGURE 11: Circuit simulation results of rotationally symmetric chaotic flow with four-wing. (a) $a=-0.05, b=1, c=0.5, R 22=1.55 \mathrm{k} \Omega$. (b) $a=-0.05, b=1, c=0.5, R 22=1.53 \mathrm{k} \Omega$.

an interesting phenomenon can be observed by changing the value of resistance $R_{22}$. For $R_{22}=1.55 \mathrm{k} \Omega$, the rotationally symmetric chaotic flow with four-wing can be observed (Figure 11(a)). Then, reducing the resistance $R_{22}$ to $R_{22}=1.53 \mathrm{k} \Omega$, the point symmetric limit cycle and axial symmetric limit cycle can be observed as shown in Figure 11(b).

\section{Conclusion}

In this study, a novel conservative chaotic system with no equilibria was investigated. Numerical analysis shows the various chaotic characteristics, including the analysis of the conservativeness, the symmetry and invariance, and coexisting chaotic behaviors. Based on one-dimensional state to feedback, a partialstate feedback controller was constructed. Then, the degenerate Hopf bifurcation method was adopted to illustrate the stable domain of control parameters. Finally, an analog circuit system was constructed to simulate the investigated chaotic system, and the circuit simulation results reproduced the numerical analysis results. Indeed, the global dynamics of this new chaotic system and the generation mechanism of chaos is still not fully explained, which is also the content of our future research.

\section{Data Availability}

The data generated or analyzed during this study are included within this article.

\section{Conflicts of Interest}

The authors declare that they have no conflicts of interest.

\section{Acknowledgments}

The authors acknowledge the referees and the editor for carefully reading this article and suggesting many helpful comments. This work was supported by the Natural Science Basic Research Program of Shaanxi (2021JM-533, 2021JQ880, and 2020JM-646), the Innovation Capability Support Program of Shaanxi (2018GHJD-21), the Science and Technology Program of Xi'an (2019218414GXRC020CG021-
GXYD20.3), and the Support Plan for Sanqin Scholars Innovation Team in Shaanxi Province of China, the Fund Project of Xijing University (XJ140231). The authors want to thank the reviewers for their insightful comments.

\section{References}

[1] E. N. Lorenz, "Deterministic nonperiodic flow," Journal of the Atmospheric Sciences, vol. 20, no. 2, pp. 130-141, 1963.

[2] O. E. Rossler, "An equation for hyperchaos," Physics Letters A, vol. 71, no. 2-3, pp. 155-157, 1979.

[3] G. Chen and T. Ueta, "Yet another chaotic attractor," International Journal of Bifurcation and Chaos, vol. 09, no. 07, pp. 1465-1466, 1999.

[4] L. Jinhu and G. Chen, "A new chaotic attractor coined," International Journal of Bifurcation and Chaos, vol. 12, no. 03, pp. 659-661, 2002.

[5] S. He, Y. Peng, and K. Sun, "Seir modeling of the covid-19 and its dynamics," Nonlinear Dynamics, vol. 101, no. 3, pp. 1667-1680, 2020.

[6] Y. Peng, S. He, and K. Sun, "A higher dimensional chaotic map with discrete memristor," AEU-International Journal of Electronics and Communications, vol. 129, Article ID 153539, 2021.

[7] C. P. Silva, "Shil'nikov's theorem-a tutorial," IEEE Transactions on Circuits and Systems I: Fundamental Theory and Applications, vol. 40, no. 10, pp. 675-682, 1993.

[8] G. A. Leonov, N. V. Kuznetsov, and V. I. Vagaitsev, "Localization of hidden Chua's attractors," Physics Letters A, vol. 375, no. 23, pp. 2230-2233, 2011.

[9] V.-T. Pham, C. Volos, S. Jafari, Z. Wei, and X. Wang, "Constructing a novel no-equilibrium chaotic system," International Journal of Bifurcation and Chaos, vol. 24, no. 5, Article ID 1450073, 2014.

[10] Z. Wang, I. Moroz, Z. Wei, and H. Ren, "Dynamics at infinity and a hopf bifurcation arising in a quadratic system with coexisting attractors," Pramana, vol. 90, no. 1, p. 12, 2018.

[11] Z. Wang, I. I. Hamarash, P. Sadeghi, and J. S. Shabestari, “A new megastable oscillator with rational and irrational parameters," International Journal of Bifurcation and Chaos, vol. 29, no. 13, 2019.

[12] X. Wang and G. Chen, "A chaotic system with only one stable equilibrium," Communications in Nonlinear Science and Numerical Simulation, vol. 17, no. 3, pp. 1264-1272, 2012. 
[13] H. Tian, Z. Wang, P. Zhang, M. Chen, and Y. Wang, "Dynamic analysis and robust control of a chaotic system with hidden attractor," Complexity, vol. 2021, Article ID 8865522, 11 pages, 2021.

[14] Z. Wang, Z. Wei, K. Sun et al., "Chaotic flows with special equilibria," The European Physical Journal Special Topics, vol. 229, no. 6-7, pp. 905-919, 2020.

[15] Z. Wang, X. Xi, L. Kong, and Z. Wei, "Infinity dynamics and ddf control for a chaotic system with one stable equilibrium," The European Physical Journal Special Topics, vol. 229, no. 6-7, pp. 1319-1333, 2020.

[16] Z. Wang, Z. Xu, E. Mliki, A. Akgul, V.-T. Pham, and S. Jafari, "A new chaotic attractor around a pre-located ring," International Journal of Bifurcation and Chaos, vol. 27, no. 10, Article ID 1750152, 2017.

[17] S. Zhang, C. Zhang, Z. Wang, and W. Kong, "Combining sparse representation and singular value decomposition for plant recognition," Applied Soft Computing, vol. 67, pp. 164-171, 2018.

[18] N. Wang, G. Zhang, N. V. Kuznetsov, and B. Han, "Hidden attractors and multistability in a modified chua's circuit," Communications in Nonlinear Science and Numerical Simulation, vol. 92, Article ID 105494, 2021.

[19] V.-T. Pham, A. Ouannas, C. Volos, and T. Kapitaniak, "A simple fractional-order chaotic system without equilibrium and its synchronization," AEU-International Journal of Electronics and Communications, vol. 86, pp. 69-76, 2018.

[20] C. Li and J. C. Sprott, "Coexisting hidden attractors in a 4-d simplified lorenz system," International Journal of Bifurcation and Chaos, vol. 24, no. 3, Article ID 1450034, 2014.

[21] A. J. Lichtenberg and M. A. Lieberman, Regular and Stochastic Motion, Springer Science \& Business Media, Berlin, Germany, 2013.

[22] S. Cang, A. Wu, Z. Wang, and Z. Chen, "Four-dimensional autonomous dynamical systems with conservative flows: twocase study," Nonlinear Dynamics, vol. 89, no. 4, pp. 2495-2508, 2017.

[23] S. Cang, Li Yue, Z. Kang, and Z. Wang, "A generic method for constructing $n$-fold covers of $3 \mathrm{~d}$ conservative chaotic systems," Chaos: An Interdisciplinary Journal of Nonlinear Science, vol. 30, no. 3, Article ID 033103, 2020.

[24] R. Guesmi, M. A. B. Farah, A. Kachouri, and M. Samet, "A novel chaos-based image encryption using dna sequence operation and secure hash algorithm sha-2," Nonlinear Dynamics, vol. 83, no. 3, pp. 1123-1136, 2016.

[25] K. Georges, E. Soujeri, and Y. Nijsure, "Design of a short reference noncoherent chaos-based communication systems," IEEE Transactions on Communications, vol. 64, no. 2, pp. 680-689, 2016.

[26] S. Jafari, V.-T. Pham, and T. Kapitaniak, "Multiscroll chaotic sea obtained from a simple $3 \mathrm{~d}$ system without equilibrium," International Journal of Bifurcation and Chaos, vol. 26, no. 02, Article ID 1650031, 2016.

[27] A. Bayani, K. Rajagopal, A. J. M. Khalaf, S. Jafari, G. D. Leutcho, and J. Kengne, "Dynamical analysis of a new multistable chaotic system with hidden attractor: antimonotonicity, coexisting multiple attractors, and offset boosting," Physics Letters A, vol. 383, no. 13, pp. 1450-1456, 2019.

[28] B. Munmuangsaen, J. Clinton Sprott, W. J.-C. Thio, A. Buscarino, and L. Fortuna, "A simple chaotic flow with a continuously adjustable attractor dimension," International Journal of Bifurcation and Chaos, vol. 25, no. 12, Article ID 1530036, 2015.
[29] Q. Lai, B. Norouzi, and F. Liu, "Dynamic analysis, circuit realization, control design and image encryption application of an extended Lü system with coexisting attractors," Chaos, Solitons \& Fractals, vol. 114, pp. 230-245, 2018.

[30] N. Wang, G. Zhang, and B. Han, "Bursting oscillations and coexisting attractors in a simple memristor-capacitor-based chaotic circuit," Nonlinear Dynamics, vol. 97, no. 4, 2019.

[31] H. Jia, W. Shi, L. Wang, and G. Qi, "Energy analysis of sprott-a system and generation of a new Hamiltonian conservative chaotic system with coexisting hidden attractors," Chaos, Solitons \& Fractals, vol. 133, Article ID 109635, 2020.

[32] C. Li and J. C. Sprott, "Variable-boostable chaotic flows," Optik, vol. 127, no. 22, pp. 10389-10398, 2016.

[33] C. Li, J. Clinton Sprott, Y. Liu, Z. Gu, and J. Zhang, "Offset boosting for breeding conditional symmetry," International Journal of Bifurcation and Chaos, vol. 28, no. 14, Article ID 1850163, 2018.

[34] T. Lu, C. Li, S. Jafari, and F. Min, "Controlling coexisting attractors of conditional symmetry," International Journal of Bifurcation and Chaos, vol. 29, no. 14, Article ID 1950207, 2019.

[35] C. Li, J. Clinton Sprott, and H. Xing, "Hypogenetic chaotic jerk flows," Physics Letters A, vol. 380, no. 11-12, pp. 1172-1177, 2016.

[36] SiC. Wang, C.H. Wang, and C. Xu, "An image encryption algorithm based on a hidden attractor chaos system and the knuth-durstenfeld algorithm," Optics and Lasers in Engineering, vol. 128, Article ID 105995, 2020.

[37] E. Dong, M. Yuan, S. Du, and Z. Chen, "A new class of Hamiltonian conservative chaotic systems with multistability and design of pseudo-random number generator," Applied Mathematical Modelling, vol. 73, pp. 40-71, 2019.

[38] H. A. Eyad and J.-H. Fu, "Local feedback stabilization and bifurcation control, i. hopf bifurcation," Systems \& Control Letters, vol. 7, no. 1, pp. 11-17, 1986.

[39] Y. Pei and G. Chen, "Hopf bifurcation control using nonlinear feedback with polynomial functions," International Journal of Bifurcation and Chaos, vol. 14, no. 05, pp. 1683-1704, 2004.

[40] D. Ding, X. Qian, W. Hu, N. Wang, and L. Dong, "Chaos and hopf bifurcation control in a fractional-order memristorbased chaotic system with time delay," The European Physical Journal Plus, vol. 132, no. 11, p. 447, 2017.

[41] C. C. Jahnke and F. E. C. Culick, "Application of bifurcation theory to the high-angle-of-attack dynamics of the $f$-14," Journal of Aircraft, vol. 31, no. 1, pp. 26-34, 1994.

[42] S. Liu, H. Ai, Z. Pang, Z. Lin, and D. Zhao, "Hopf bifurcation control of nonlinear electromechanical coupling main drive system of rolling mill," The European Physical Journal Plus, vol. 135, no. 4, p. 365, 2020.

[43] D. Hu and H. Cao, "Bifurcation and chaos in a discrete-time predator-prey system of Holling and Leslie type," Communications in Nonlinear Science and Numerical Simulation, vol. 22, no. 1-3, pp. 702-715, 2015.

[44] N. Shuichi, "Constant temperature molecular dynamics methods," Progress of Theoretical Physics Supplement, vol. 103, pp. 1-46, 1991.

[45] C. Li, J. Sun, T. Lu, and T. Lei, "Symmetry evolution in chaotic system," Symmetry, vol. 12, no. 4, p. 574, 2020.

[46] C. Li and J. Clinton Sprott, "Multistability in the lorenz system: a broken butterfly," International Journal of Bifurcation and Chaos, vol. 24, no. 10, Article ID :1450131, 2014.

[47] J. Sotomayor, L. Fernando Mello, and B. Denis de Carvalho, "Bifurcation analysis of the watt governor system," Computational \& Applied Mathematics, vol. 26, no. 1, pp. 19-44, 2007. 